\title{
The effects of midazolam or propofol plus fentanyl on ICU mortality: a retrospective study based on the MIMIC-IV database
}

\author{
Wen Sun ${ }^{1,2 \#}$, Yang Yan ${ }^{3 \#}$, Shidong $\mathrm{Hu}^{3}$, Boyan Liu ${ }^{3}$, Shuying Wang ${ }^{4}$, Wenli $\mathrm{Yu}^{5}$, Songyan $\mathrm{Li}^{3}$ \\ ${ }^{1}$ The First Central Clinical School, Tianjin Medical University, Tianjin, China; ${ }^{2}$ Department of Anesthesiology, the Second Affiliated Hospital \\ of Tianjin University of Traditional Chinese Medicine, Tianjin, China; ${ }^{3}$ Department of General Surgery, the First Medical Center, Chinese PLA \\ General Hospital, Beijing, China; ${ }^{4}$ Department of Blood Transfusion, the First Medical Center, Chinese PLA General Hospital, Beijing, China; \\ ${ }^{5}$ Department of Anesthesiology, Tianjin First Central Hospital, Tianjin, China \\ Contributions: (I) Conception and design: W Sun, Y Yan; (II) Administrative support: S Hu, B Liu; (III) Provision of study materials or patients: B Liu, \\ S Wang; (IV) Collection and assembly of data: W Sun, Y Yan, S Hu, B Liu, S Wang; (V) Data analysis and interpretation: W Sun, Y Yan, S Li, W Yu; \\ (VI) Manuscript writing: All authors; (VII) Final approval of manuscript: All authors. \\ \#These authors contributed equally to this work. \\ Correspondence to: Wenli Yu. Department of Anesthesiology, Tianjin First Central Hospital, Tianjin, China. Email: yzxyuwenli@163.com; Songyan Li. \\ Department of General Surgery, the First Medical Center, Chinese PLA General Hospital, Beijing, China. Email: lisongyan301@163.com.
}

Background: Patients in the intensive care unit (ICU) are often under stress and fail to cooperate well with invasive treatments. Analgesia and sedation are of great significance for reducing the suffering of patients and ensuring the application and effectiveness of treatment. For better clinical choice, we aimed to explore the effect of the combination of propofol + fentanyl or midazolam + fentanyl on the short-term prognosis of hospitalized patients in the ICU.

Methods: According to the inclusion and exclusion criteria, we retrospectively included patients in the MIMIC-IV database receiving midazolam + fentanyl or propofol + fentanyl analgesic and sedative treatment using Structured Query Language (SQL) to extract clinical data from the MIMIC-IV database. The primary endpoint was the death rate within 28 days after the patient was admitted to the ICU. Doubly robust estimation was used to infer the relationship between sedation and analgesia and 28 days outcome. The gradient boosted model (GBM) was used to estimate the propensity score (PS) of the patient's sedation and analgesia program, PS was used as the weight, and the inverse probabilities weighting (IPW) model was used to generate a weighted cohort.

Results: In total, 4,188 cases were included, with 2,174 (51.9\%) in the propofol group and 2,014 (48.1\%) in the midazolam group. In the PS score matching cohort, the 28-day mortality of patients in the midazolam group was $30.8 \%$, and the 28 -day mortality of patients in the propofol group was $25.5 \%$. The adjusted odds ratio $(\mathrm{OR})$ value was 1.421 [95\% confidence interval (CI): 1.118-1.806, $\mathrm{P}<0.001$ ]. Patients in the propofol group did not use vasoactive drugs for a longer period of time than the midazolam group, and patients in the propofol group received significantly more fluids than those in the midazolam group in the first three days after admission to the ICU.

Conclusions: Compared with midazolam combined with fentanyl, propofol combined with fentanyl for sedation and analgesia can reduce the risk of short-term death in ICU patients.

Keywords: Intensive care unit; propofol; midazolam; fentanyl; death

Submitted Dec 09, 2021. Accepted for publication Feb 21, 2022.

doi: $10.21037 /$ atm-22-477

View this article at: https://dx.doi.org/10.21037/atm-22-477 


\section{Introduction}

Severely ill patients are exposed to intense stress upon admission to the intensive care unit (ICU) or after admission but in a repeated and continuous stress environment for a period of time (1-3). Common causes of stress include: (I) the patient's severe trauma or serious illness, which may lead to severe pain or impaired organ function; (II) environmental factors: the ICU ward is often illuminated for 24 hours, the patient does not have a three-meal rhythm, and the biological clock is disrupted. Meanwhile, there are various noises (machine sounds, alarms, shouts), as well as the rescue or death of patients in the same room, which, combined with their condition, can lead to a certain degree of sleep deprivation; (III) occult pain and various tubes (such as tracheal intubation, urinary catheters, gastric tubes, and various drainage tubes) cause trauma and pain to the corresponding parts, and long-term bed rest causes pain in the compressed region; (IV) fear, helplessness, and misses caused by the condition $(2,3)$. For these reasons, severely ill patients tend to be under intense stress, triggering adverse reactions, such as irritability, resistance, anxiety, and depression, which may cause further deterioration of the condition and severely affect the treatment outcome (4). Therefore, many guidelines currently recommend that analgesia and sedation should be used as routine treatments for ICU patients, and it is recommended that appropriate analgesia be given at the same time or before sedation (5-7). Therefore, in ICU patients, analgesics and sedatives are often used in combination.

Previous study has shown that the use of different analgesic and sedative drugs has a variety of adverse reactions, especially for patients who already have consciousness disturbances and decreased cardiopulmonary function, which may not reduce short-term mortality but increase the risk of adverse reactions (8). In an openlabel, randomized study, Shehabi et al. found that early use of dexmedetomidine in ICU patients did not reduce the 90-day mortality rate, but the incidence of adverse reactions increased significantly (8). Therefore, a reasonable choice of sedation and analgesia is needed to reduce the incidence of adverse reactions. In the ICU, commonly used analgesics are divided into opioids and nonopioids. The commonly used sedatives include benzodiazepines, propofol and dexmedetomidine. In clinical practice, various combination schemes, such as propofol + fentanyl or midazolam + fentanyl, are often used, but the impact of these schemes on the short-term prognosis of severely ill patients is not currently clear, as study results have been inconsistent. The MIMIC-IV database is an open critical medicine database (9) jointly released by the Computational Physiology Laboratory of the Massachusetts Institute of Technology, Beth Israel Dikang Medical Center and Philips Medical under the funding of the National Institutes of Health (9). The latest version is currently MIMIC-IV. The database contains clinical real-world data of more than 60,000 critically ill patients and is an important resource for retrospective research. The purpose of this study was to retrospectively analyze patient data in the MIMICIV database to explore the impact of the combination of propofol + fentanyl or midazolam + fentanyl on the shortterm prognosis of hospitalized ICU patients.

We present the following article in accordance with the STROBE reporting checklist (available at https://atm. amegroups.com/article/view/10.21037/atm-22-477/rc).

\section{Methods}

\section{Study population}

This study was a retrospective study, and the research subjects were adult patients admitted to the ICU. Inclusion criteria included: (I) age $\geq 18$ years; (II) first admission to the ICU (for patients who had been admitted to the ICU multiple times, only the first-time data were collected); (III) stay in the ICU $\geq 24 \mathrm{~h}$; (IV) after admission to the ICU, they received sedation + analgesia; (V) propofol or midazolam was used for sedation, and fentanyl was used for analgesia. Exclusion criteria included: (I) absence of important data, such as sequential organ failure assessment (SOFA) and simplified acute physiological score I (SAPS I); (II) lost to follow-up; (III) other sedation or analgesic drugs were used. All patient-related information in the MIMICIV database was anonymous, and informed consent was not required. The study was conducted in accordance with the Declaration of Helsinki (as revised in 2013).

\section{Data source}

The data of this study came from the MIMIC-IV database (https://mimic-iv.mit.edu/). The database collected hospitalization information of more than 60,000 patients admitted to the ICU of the Beth Israel Dikang Medical Center in the United States. Data included vital signs, medications, laboratory measurements, observations, records drawn by nursing staff, fluid balance, program 
codes, diagnostic codes, imaging reports, hospital stay and survival data.

\section{Data extraction}

We used Structured Query Language (SQL) to extract the target data from the MIMIC-IV database for current analysis and propensity score matching analysis: age; gender; body weight; white blood cell count; hemoglobin; platelet count; blood urea nitrogen; blood creatinine; blood glucose; blood electrolytes including $\mathrm{K}^{+}, \mathrm{Na}^{+}, \mathrm{Ca}^{2+}$; blood gas test results such as $\mathrm{HCO}_{3}{ }^{-}$; sequential organ failure score (SOFA); length of stay in the ICU; and death in the ICU; and information of comorbidities, including hypertension, coronary heart disease, chronic obstructive pulmonary disease, chronic kidney disease, and whether mechanical surgery was performed during ICU hospitalization. Ventilation, use of vasoactive drugs and renal replacement therapy, whether complicated by ventilator-related pneumonia, urinary tract infection, diabetic ketoacidosis, acute myocardial infarction, sedation, analgesic drugs were also measured. These data were all important indicators for ICU patients. Among these data, SOFA is an important indicator for assessing the likelihood and severity of sepsis. length of stay in the ICU and death were both important outcomes for studies on ICU patients. All laboratory test parameters were extracted from the results acquired within the first 24 hours after admission to the ICU (i.e., the baseline value) and the extreme values during the ICU stay period (i.e., the maximum value and minimum value).

\section{Outcome}

The primary endpoint was mortality within 28 days after the admission to the ICU. Secondary endpoints included the number of days of without mechanical ventilation during 28 days after admission to the ICU, the number of days without vasoactive drug use, the maximum dose of norepinephrine, the fluid input volume of the patient in the first three days after admission to the ICU, the level and the rate of decrease of serum lactic acid, and the rate of decrease of creatinine. Calculation of the decrease in lactic acid and creatinine: for patients receiving sedation and analgesia, the last test result before treatment was used to subtract the first and second test results 24 hours and 48 hours after the end of treatment.

\section{Statistical analysis}

SPSS software (version 23.0, IBM, Chicago, USA) was used to perform statistical analysis on all research data. Continuous variables were first tested for normality of distribution. If normally distributed, they were expressed as the means (standard deviation). The comparison between the two groups was conducted with an independent sample $t$-test; if they were nonnormally distributed, the median $M$ (QL, QU) was used, and the Wilcoxon rank sum test was used for comparison between the two groups. Categorical variables were expressed as the number and percentages ( $\mathrm{n}, \%)$, and the comparisons between the two groups was conducted using $\chi^{2}$. Multivariate logistic regression analysis was used to analyze the risk factors related to death of patients within 28 days of admission to the ICU, and the variables that remained unbalanced between the groups with and without a TTE in the propensity score model were adjusted. Doubly robust estimation was used to infer the relationship between sedation and analgesia and patient endpoint events (10). The gradient boosted model (GBM) was used to estimate the propensity score (PS) of the patient's sedation and analgesia program to minimize the imbalance of variables between the two sedation and analgesia programs. With PS as the weight, a weighted queue was generated using an inverse probability weighting (IPW) model (11). A two-sided $\mathrm{P}<0.05$ indicated a statistically significant difference.

\section{Results}

\section{General information}

According to the inclusion and exclusion criteria, 4188 cases were extracted from the MIMIC-IV database (Figure 1) and divided into a propofol group and a midazolam group. There were 2,174 cases $(51.91 \%)$ in the propofol group, $913(42.00 \%)$ of which were female, and 2,014 cases $(48.09 \%)$ in the midazolam group, $856(42.50 \%)$ of which were female. The SAPS score $(20.04 \pm 4.85$ vs. $21.13 \pm 5.70, \mathrm{P}<0.001)$, SOFA score $(5.10 \pm 3.35$ vs. $6.25 \pm 4.03$, $\mathrm{P}<0.001)$ and Elixhauser score $(6.89 \pm 7.96$ vs. $11.20 \pm 9.12$, $\mathrm{P}<0.001)$ of patients in the propofol group were lower than those in the midazolam group. The rate of patients receiving mechanical ventilation was significantly higher in the propofol group than in the midazolam group (99.60\% vs. $76.90 \%, \mathrm{P}<0.001$ ), and there was no significant 


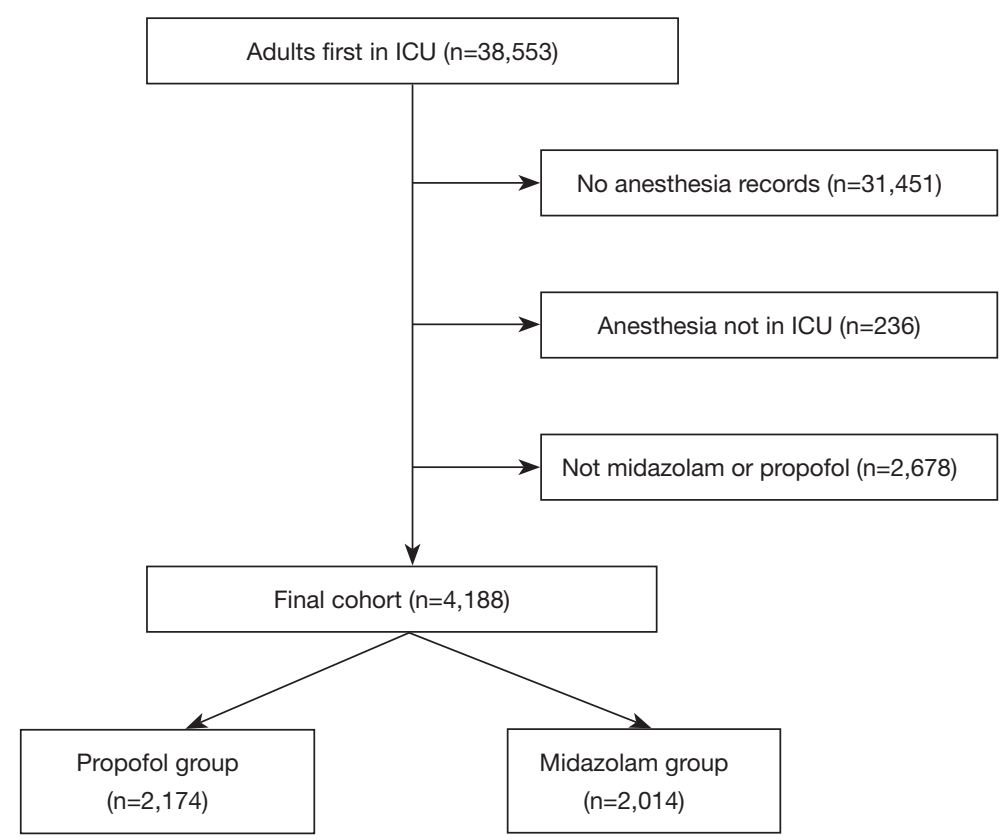

Figure 1 Flowchart of cases enrollment. ICU, intensive care unit.

difference in the rate of vasoactive medication $39.40 \%$ vs. $40.80 \%, \mathrm{P}=0.358)$. The rate of stroke was higher than that of the midazolam group, and the rates of chronic heart failure, atrial fibrillation, decreased renal function, liver function impairment, chronic obstructive pulmonary disease, coronary heart disease and malignant tumors were lower in the propofol group than in the midazolam group (Table 1). For propensity score matching analysis, each group included 702 patients (Table 1).

\section{Double robust analysis}

The results of PS analysis using GBM based on covariates are shown in Figure 2. From the figure, we can see the contribution of different covariates to the PS score. In this study, the covariates that had a greater contribution to PS included the use of ventilators, partial pressure of oxygen $\left(\mathrm{PO}_{2}\right)$, blood urea nitrogen, troponin, and Elixhauser score. Based on this result, IPW was used to standardize the differences between the propofol and midazolam groups (Table 1). Many of the indicators were not significantly different between the two groups based on the PS score. The SAPS score, central venous pressure, blood BUN level and creatinine level were higher in the propofol group than in the midazolam group, while the hemoglobin level and oxygen pressure were lower than those in the midazolam group (Table 1). Further regression analysis adjusted the variables that were different between groups.

\section{Endpoint analysis}

Double robust analysis showed that the propofol group had lower risk of death within 28 days after admission to the ICU than the midazolam group. In the PS score compatibility cohort, the 28-day mortality of patients was $30.8 \%$ and $25.5 \%$ in the midazolam group and the propofol group, respectively. The adjusted odds ration (OR) value was 1.421 [95\% confidence interval (CI): 1.118-1.806, $\mathrm{P}<0.001]$. Similarly, the analysis results of the other four models were consistent, suggesting that propofol was beneficial to reducing the risk of death during 28 days ICU stay (Table 2). The analysis of the secondary endpoints showed that patients in the propofol group did not use vasoactive drugs for a longer period of time than the midazolam group and that patients in the propofol group had significantly more fluid input than mimics in the first three days after admission to the ICU. For patients in the midazolam group, other secondary endpoints were not significantly different between the two groups (Table 3).

\section{Discussion}

Based on the MIMIC-IV database, this study retrospectively analyzed the effects of the propofol + fentanyl regimen and 
Table 1 Comparison of characteristics between the original cohort and the adjusted (weighted) cohort

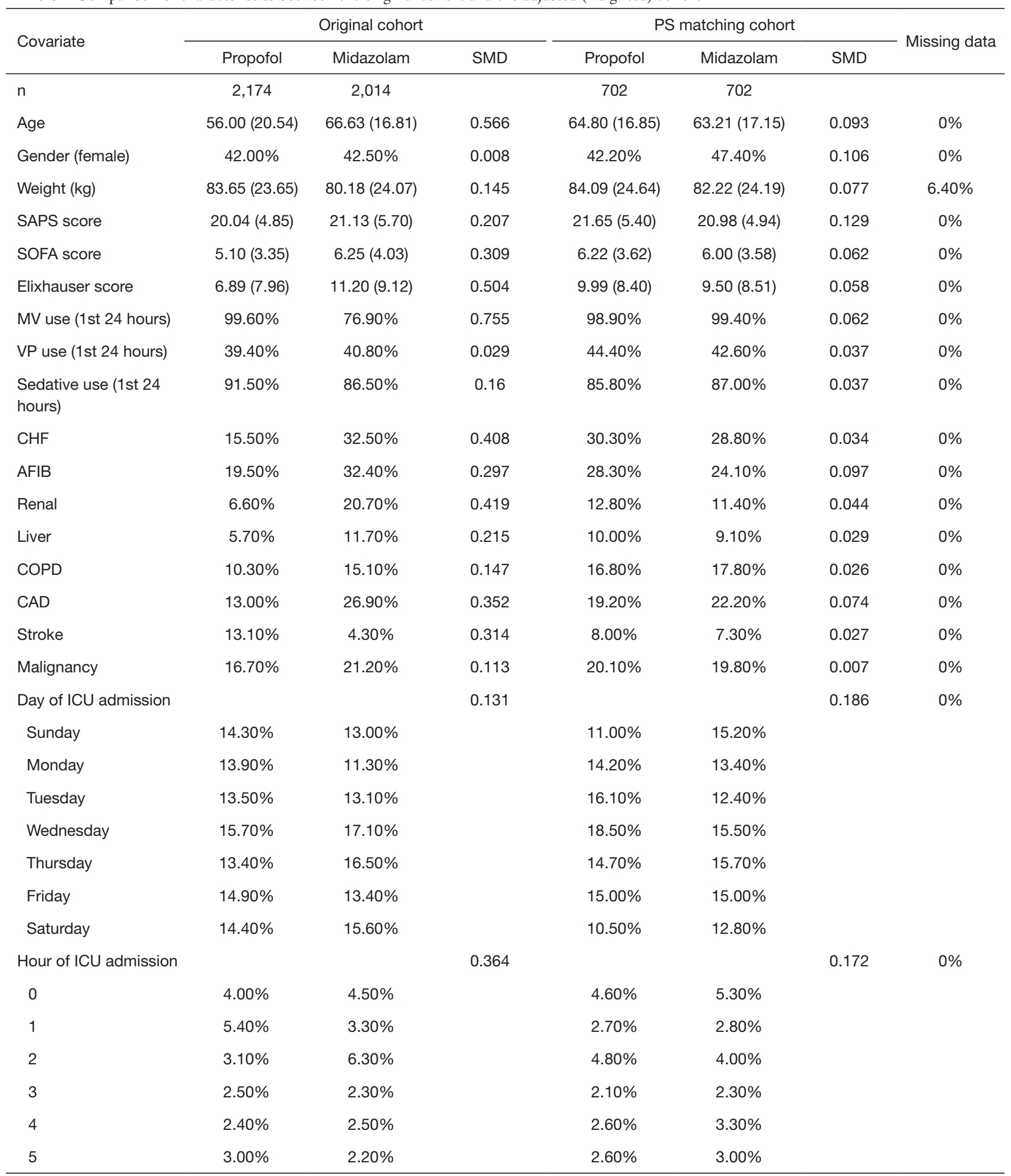

Table 1 (continued) 
Table 1 (continued)

\begin{tabular}{|c|c|c|c|c|c|c|c|}
\hline Covariate & \multicolumn{3}{|c|}{ Original cohort } & \multicolumn{3}{|c|}{ PS matching cohort } & Missing data \\
\hline 6 & $2.00 \%$ & $2.20 \%$ & & $2.10 \%$ & $2.60 \%$ & & \\
\hline 7 & $1.50 \%$ & $1.60 \%$ & & $1.70 \%$ & $1.70 \%$ & & \\
\hline 8 & $2.20 \%$ & $1.80 \%$ & & $1.60 \%$ & $2.60 \%$ & & \\
\hline 10 & $4.30 \%$ & $2.10 \%$ & & $2.40 \%$ & $3.00 \%$ & & \\
\hline 11 & $4.00 \%$ & $3.60 \%$ & & $4.30 \%$ & $4.80 \%$ & & \\
\hline 12 & $3.80 \%$ & $7.20 \%$ & & $3.80 \%$ & $4.60 \%$ & & \\
\hline 13 & $3.30 \%$ & $2.70 \%$ & & $2.80 \%$ & $3.60 \%$ & & \\
\hline 16 & $4.70 \%$ & $5.30 \%$ & & $5.70 \%$ & $5.80 \%$ & & \\
\hline 17 & $5.10 \%$ & $5.00 \%$ & & $5.80 \%$ & $5.00 \%$ & & \\
\hline 18 & $6.00 \%$ & $4.20 \%$ & & $5.70 \%$ & $4.70 \%$ & & \\
\hline 19 & $9.40 \%$ & $5.80 \%$ & & $5.80 \%$ & $7.00 \%$ & & \\
\hline 20 & $6.00 \%$ & $6.20 \%$ & & $6.80 \%$ & $6.60 \%$ & & \\
\hline 21 & $5.80 \%$ & $9.10 \%$ & & $6.40 \%$ & $5.70 \%$ & & \\
\hline 22 & $4.60 \%$ & $6.00 \%$ & & $6.30 \%$ & $5.10 \%$ & & \\
\hline 23 & $4.90 \%$ & $5.00 \%$ & & $6.30 \%$ & $4.80 \%$ & & \\
\hline WBC $\left(\times 10^{9}\right)$ & $12.93(7.99)$ & $14.09(15.25)$ & 0.095 & $13.82(10.24)$ & $13.71(8.62)$ & 0.012 & $2.40 \%$ \\
\hline Hemoglobin (g/L) & $11.21(2.27)$ & $10.73(2.21)$ & 0.214 & $10.69(2.15)$ & $10.92(2.13)$ & 0.105 & $2.30 \%$ \\
\hline Platelet $\left(\times 10^{9}\right)$ & 219.17 (113.76) & $223.10(133.61)$ & 0.032 & 219.67 (128.19) & $231.73(130.82)$ & 0.093 & $2.30 \%$ \\
\hline Sodium (mmol/L) & $138.69(4.94)$ & $139.20(6.23)$ & 0.091 & $138.37(5.48)$ & $138.53(5.23)$ & 0.03 & $1.60 \%$ \\
\hline Potassium (mmol/L) & $4.08(0.85)$ & $4.23(0.81)$ & 0.175 & $4.21(0.76)$ & $4.13(0.77)$ & 0.112 & $1.60 \%$ \\
\hline Bicarbonate $(\mathrm{mmHg})$ & $22.95(4.31)$ & $22.35(5.59)$ & 0.12 & $22.30(5.36)$ & $22.38(5.27)$ & 0.014 & $1.90 \%$ \\
\hline Chloride (mmol/L) & $106.39(5.91)$ & $106.20(7.38)$ & 0.028 & $105.52(6.68)$ & $105.73(6.57)$ & 0.031 & $1.70 \%$ \\
\hline Bun (mmol/L) & $20.75(17.10)$ & $35.14(27.62)$ & 0.626 & 30.89 (23.72) & 26.21 (19.94) & 0.214 & $1.90 \%$ \\
\hline Lactate $(\mathrm{mmol} / \mathrm{L})$ & $2.98(2.28)$ & $2.72(2.53)$ & 0.109 & $2.90(2.64)$ & $2.80(2.59)$ & 0.038 & $28.50 \%$ \\
\hline Creatinine (mmol/L) & $1.14(1.06)$ & $1.63(1.59)$ & 0.362 & $1.58(1.47)$ & $1.37(1.29)$ & 0.155 & $1.90 \%$ \\
\hline $\mathrm{PH}$ & $7.36(0.09)$ & $7.34(0.12)$ & 0.19 & $7.34(0.11)$ & $7.34(0.10)$ & 0.009 & $16.50 \%$ \\
\hline
\end{tabular}

Table 1 (continued) 
Table 1 (continued)

\begin{tabular}{|c|c|c|c|c|c|c|c|}
\hline Covariate & \multicolumn{3}{|c|}{ Original cohort } & \multicolumn{3}{|c|}{ PS matching cohort } & Missing data \\
\hline $\mathrm{PO}_{2}(\mathrm{mmHg})$ & 202.54 (121.93) & $154.04(123.12)$ & 0.396 & $160.54(110.52)$ & $173.04(112.73)$ & 0.112 & $17.70 \%$ \\
\hline $\mathrm{PCO}_{2}(\mathrm{mmHg})$ & 41.55 (10.39) & $42.58(14.13)$ & 0.083 & 42.77 (13.69) & 42.04 (11.32) & 0.058 & $17.70 \%$ \\
\hline BNP (tested) & $0.50 \%$ & $2.30 \%$ & 0.152 & $1.10 \%$ & $2.10 \%$ & 0.079 & $0 \%$ \\
\hline
\end{tabular}

MV, mechanical ventilation; VP, vasopressor; SAPS, simplified acute physiological score; SOFA, sequential organ failure score; CHF, chronic heart failure; AFIB, atrial fibrillation; COPD, chronic obstructive pulmonary disease; CAD, coronary artery disease; ICU, intensive care unit; CVP, central venous pressure; WBC, white blood cell; BUN, blood urea nitrogen; BNP, brain natriuretic peptide; SMD, standardized mean differences; MAP, mean arterial pressure.

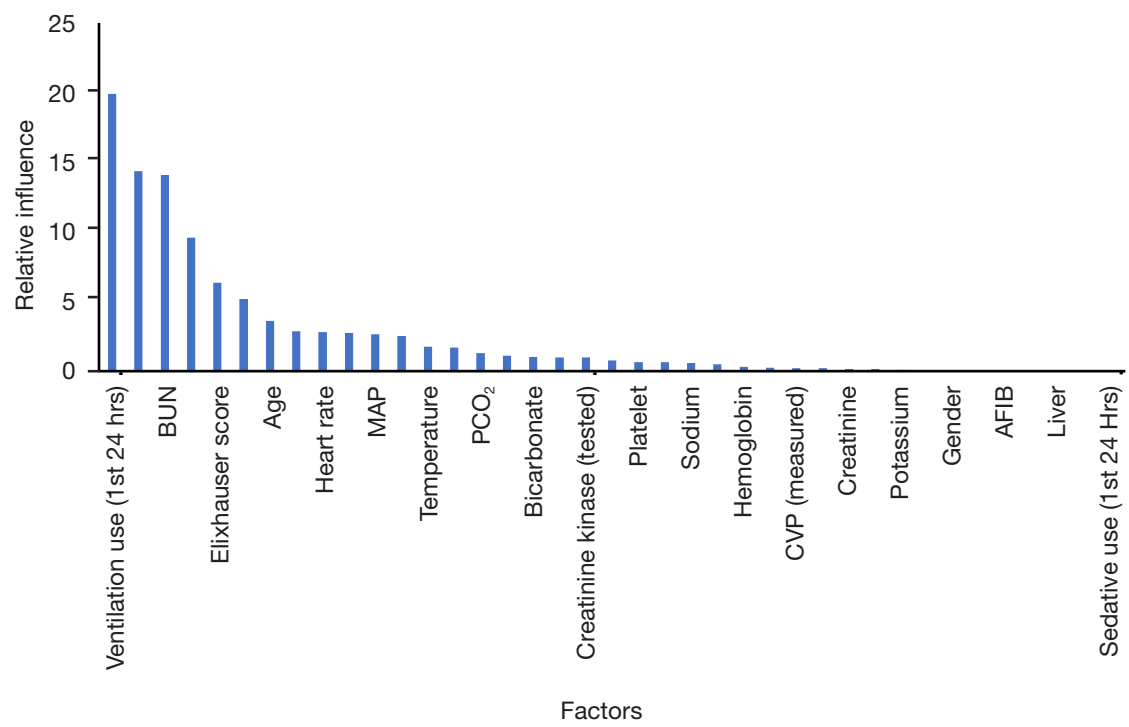

Figure 2 Relative influence factor of covariates. The relative influence factor measures how discriminative the 40 covariates of the propensity score model were when predicting the likelihood of echocardiogram performance. BUN, blood urea nitrogen; MAP, mean arterial pressure; CVP, central venous pressure; AFIB, atrial fibrillation.

midazolam + fentanyl regimen on the short-term prognosis of hospitalized patients in the ICU. Compared with midazolam combined with fentanyl, propofol combined with fentanyl reduced the 28-day risk of death in severely ill patients in the ICU. Further analysis revealed that the fluid input in the first three days after admission to the ICU was significantly higher in the propofol group than in the midazolam group, and the period of time without the use of vasoactive drugs was longer. There were no significant differences in the time of mechanical ventilation, dopamine use rate, SOFA score, norepinephrine measurement, or changes in serum lactic acid levels during the treatment process between the two groups.

Most patients in the intensive care unit require mechanical ventilation and other invasive treatments. However, these treatments themselves can cause stress, pain, discomfort, and even complications (12). Ensuring the effectiveness of mechanical ventilation and other treatments and improving the comfort of patients in the ICU are the main purposes of analgesia and sedation treatment, and it is also an important measure to improve the safety of the treatment process $(13,14)$. However, analgesia and sedation therapy itself may also increase the risk of death and the risk of complications for patients. Some researchers 
Table 2 Primary outcome analysis with five different models

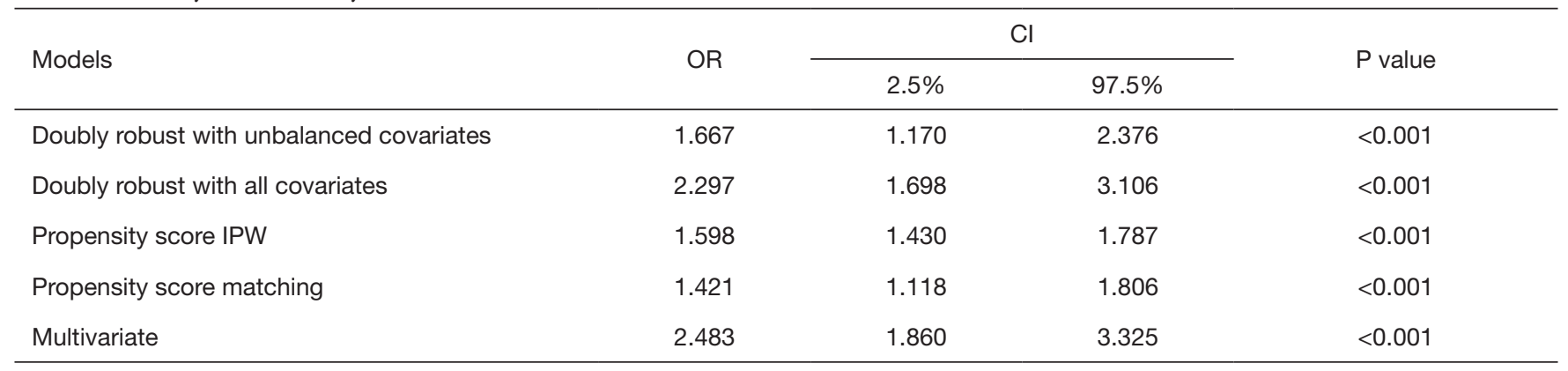

OR, odds ratio; $\mathrm{Cl}$, confidence interval; IPW, inverse probabilities weighting.

Table 3 Secondary outcome analysis with propensity score-matched cohorts

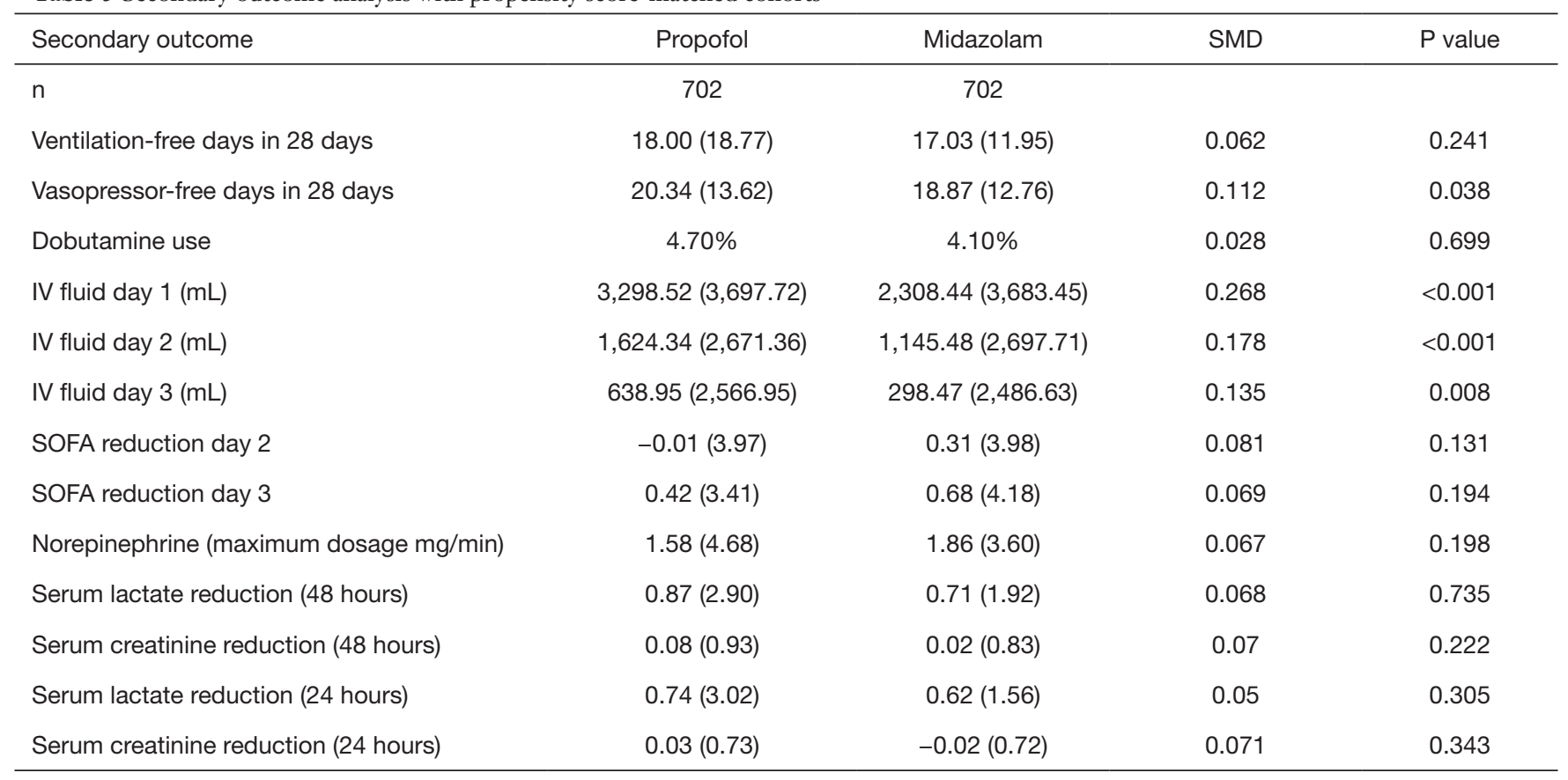

SMD, standardized mean differences; IV, intravenous; SOFA sequential organ failure score.

worry that analgesia and sedation treatment will prolong the time of mechanical ventilation and ICU hospital stay. For this reason, a study found that no sedation treatment plan cannot reduce the 90 -day mortality rate of patients receiving mechanical ventilation in the ICU (15). In this multicenter randomized controlled study, the researchers included 700 critically ill patients in a 1:1 ratio and divided them into a nonsedation group and a light sedation group. The results showed that the 90-day fatality rates of patients in the nonsedation group and the light sedation group were $42.4 \%$ and $37.0 \%$, respectively, with a difference of $5.4 \%$ (95\% CI: $-2.2 \%-12.2 \%, \mathrm{P}=0.65$ ). Meanwhile, there were no significant differences in the number of days that the two groups of patients were admitted to the ICU or the number of days on ventilator-assisted ventilation (15).

When choosing analgesia and sedation treatment plans, doctors should formulate individualized plans based on the patient's state and monitor the patient's response to the drug to make appropriate adjustments (16). In clinical practice, many types of drugs can be used for analgesia and sedation in the ICU. At present, the commonly used analgesics in clinical practice are mainly fentanyl, remifentanil and sufentanil, as well as nonopioid drugs, such as katamine, carbamezapine, gabapentin and paracetamol. Commonly 
used sedative drugs are mainly propofol, dexmedetomidine, and midazolam. These analgesics and sedatives can be used in combination. Previous study has shown that propofol, midazolam, and dexmedetomidine have similar sedative effects on severely ill patients in the ICU, but midazolam is associated with longer mechanical ventilation times (17). Metomidine increases the risk of adverse reactions (17). Similar study has also found that, for patients with sepsis in the ICU, the use of dexmedetomidine during mechanical ventilation did not reduce mortality or shorten the duration of mechanical ventilation (18). Propofol is safe and effective and is widely used in clinical sedative treatment. It has the characteristics of quick recovery and better sedative and forgetting effects; compared with other traditional sedatives, it does not increase the incidence of cardiopulmonary complications (19). Propofol is widely used for sedative therapy in the ICU (20). Many studies have shown that, compared with midazolam, the prognosis of severely ill patients treated with propofol is better, including the duration of mechanical ventilation and tracheal removal in the ICU. Tube time is shorter $(21,22)$, and propofol is associated with lower medical costs (23). This study included real-world cases with a large sample size, but the results were inconsistent with previous studies. The analysis results did not show that severely ill propofoltreated ICU patients had shorter mechanical ventilation times and shorter tracheal extubation times. This may be related to the retrospective nature of the studies. In real-world clinical practice, doctors may not pay much attention to the time of mechanical ventilation and the time of extubation, as in randomized controlled studies. That is, in randomized controlled studies, doctors may be concerned about mechanical ventilation. More attention is given to the time of tracheal extubation, and the assessment is timelier. However, the results of this study show that the short-term mortality rate of ICU patients treated with propofol was lower. From this point of view, it is consistent with the results of previous studies; that is, compared with midazolam, propofol can improve the short-term prognosis of critically ill patients in the ICU.

Limitations of this study: although this study retrospectively included a large sample size of realworld cases for analysis, it still has inherent limitations of retrospective research. The analgesia and sedation regimens used by these patients may be related to the experience and tendencies of doctors and medical institutions, which may have caused bias in case selection. Second, some patients were excluded due to incomplete data, resulting in a decrease in the representativeness of the research samples. At the same time, unlike other studies, the primary endpoint of this study was death 28 days after admission to the ICU, while the endpoint of other previous studies was death at 90 days. Therefore, this study lacks an assessment of the 90-day outcome.

\section{Acknowledgments}

Funding: None.

\section{Footnote}

Reporting Checklist: The authors have completed the STROBE reporting checklist. Available at https://atm. amegroups.com/article/view/10.21037/atm-22-477/rc

Conflicts of Interest: All authors have completed the ICMJE uniform disclosure form (available at https://atm. amegroups.com/article/view/10.21037/atm-22-477/coif). The authors have no conflicts of interest to declare.

Ethical Statement: The authors are accountable for all aspects of the work in ensuring that questions related to the accuracy or integrity of any part of the work are appropriately investigated and resolved. The study was conducted in accordance with the Declaration of Helsinki (as revised in 2013).

Open Access Statement: This is an Open Access article distributed in accordance with the Creative Commons Attribution-NonCommercial-NoDerivs 4.0 International License (CC BY-NC-ND 4.0), which permits the noncommercial replication and distribution of the article with the strict proviso that no changes or edits are made and the original work is properly cited (including links to both the formal publication through the relevant DOI and the license). See: https://creativecommons.org/licenses/by-nc-nd/4.0/.

\section{References}

1. Hatch R, Young D, Barber V, et al. Anxiety, Depression and Post Traumatic Stress Disorder after critical illness: a UK-wide prospective cohort study. Crit Care 2018;22:310.

2. Lusk B, Lash AA. The stress response, psychoneuroimmunology, and stress among ICU patients. Dimens Crit Care Nurs 2005;24:25-31.

3. Davydow DS, Gifford JM, Desai SV, et al. Posttraumatic 
stress disorder in general intensive care unit survivors: a systematic review. Gen Hosp Psychiatry 2008;30:421-34.

4. Garrouste-Orgeas M, Flahault C, Vinatier I, et al. Effect of an ICU Diary on Posttraumatic Stress Disorder Symptoms Among Patients Receiving Mechanical Ventilation: A Randomized Clinical Trial. JAMA 2019;322:229-39.

5. Hinkelbein J, Lamperti M, Akeson J, et al. European Society of Anaesthesiology and European Board of Anaesthesiology guidelines for procedural sedation and analgesia in adults. Eur J Anaesthesiol 2018;35:6-24.

6. Critical Care Medicine Branch of Chinese Medical Association. Chinese Adult ICU Analgesia and Sedative Treatment Guidelines. Chinese Critical Care Medicine 2018;30:497-514.

7. Barr J, Fraser GL, Puntillo K, et al. Clinical practice guidelines for the management of pain, agitation, and delirium in adult patients in the intensive care unit. Crit Care Med 2013;41:263-306.

8. Shehabi Y, Howe BD, Bellomo R, et al. Early Sedation with Dexmedetomidine in Critically Ill Patients. N Engl J Med 2019;380:2506-17.

9. Johnson AE, Pollard TJ, Shen L, et al. MIMIC-III, a freely accessible critical care database. Sci Data 2016;3:160035.

10. Dukes O, Martinussen T, Tchetgen Tchetgen EJ, et al. On doubly robust estimation of the hazard difference. Biometrics 2019;75:100-9.

11. Matsouaka RA, Atem FD. Regression with a rightcensored predictor using inverse probability weighting methods. Stat Med 2020;39:4001-15.

12. Australian PIft, New Zealand Intensive Care Society Clinical Trials Group AHSCCSCN, the Irish Critical Care Trials G, et al. Effect of Stress Ulcer Prophylaxis With Proton Pump Inhibitors vs Histamine-2 Receptor Blockers on In-Hospital Mortality Among ICU Patients Receiving Invasive Mechanical Ventilation: The PEPTIC Randomized Clinical Trial. JAMA 2020;323:616-26.

13. Vagionas D, Vasileiadis I, Rovina N, et al. Daily

Cite this article as: Sun W, Yan Y, Hu S, Liu B, Wang S, Yu W, $\mathrm{Li}$ S. The effects of midazolam or propofol plus fentanyl on ICU mortality: a retrospective study based on the MIMIC-IV database. Ann Transl Med 2022;10(4):219. doi: 10.21037/atm$22-477$ sedation interruption and mechanical ventilation weaning: a literature review. Anaesthesiol Intensive Ther 2019;51:380-9.

14. Pearson SD, Patel BK. Evolving targets for sedation during mechanical ventilation. Curr Opin Crit Care 2020;26:47-52.

15. Olsen HT, Nedergaard HK, Strom T, et al. Nonsedation or Light Sedation in Critically Ill, Mechanically Ventilated Patients. N Engl J Med 2020;382:1103-11.

16. Wiatrowski R, Norton C, Giffen D. Analgosedation: Improving Patient Outcomes in ICU Sedation and Pain Management. Pain Manag Nurs 2016;17:204-17.

17. Jakob SM, Ruokonen E, Grounds RM, et al. Dexmedetomidine vs midazolam or propofol for sedation during prolonged mechanical ventilation: two randomized controlled trials. JAMA 2012;307:1151-60.

18. Kawazoe Y, Miyamoto K, Morimoto T, et al. Effect of Dexmedetomidine on Mortality and Ventilator-Free Days in Patients Requiring Mechanical Ventilation With Sepsis: A Randomized Clinical Trial. JAMA 2017;317:1321-8.

19. Eleveld DJ, Colin P, Absalom AR, et al. Pharmacokineticpharmacodynamic model for propofol for broad application in anaesthesia and sedation. Br J Anaesth 2018;120:942-59.

20. Mirenda J, Broyles G. Propofol as used for sedation in the ICU. Chest 1995;108:539-48.

21. Garcia R, Salluh JIF, Andrade TR, et al. A systematic review and meta-analysis of propofol versus midazolam sedation in adult intensive care (ICU) patients. J Crit Care 2021;64:91-9.

22. Hall RI, Sandham D, Cardinal P, et al. Propofol vs midazolam for ICU sedation : a Canadian multicenter randomized trial. Chest 2001;119:1151-9.

23. Barrientos-Vega R, Mar Sanchez-Soria M, Morales-Garcia $\mathrm{C}$, et al. Prolonged sedation of critically ill patients with midazolam or propofol: impact on weaning and costs. Crit Care Med 1997;25:33-40. 